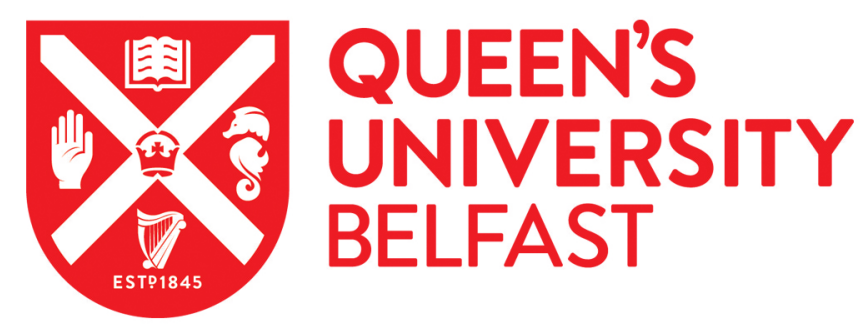

\title{
System service provision capabilities of storage devices connected to a MV distribution network: A Northern Ireland case study
}

Bagchi, A., Best, R., Morrow, D. J., Pollock, J., Bailie, I., \& Cupples, A. (2020). System service provision capabilities of storage devices connected to a MV distribution network: A Northern Ireland case study. In 2020 IEEE Power and Energy Society Innovative Smart Grid Technologies Conference, ISGT 2020 (2020 IEEE Power and Energy Society Innovative Smart Grid Technologies Conference, ISGT 2020). Institute of Electrical and Electronics Engineers Inc.. https://doi.org/10.1109/ISGT45199.2020.9087783

Published in:

2020 IEEE Power and Energy Society Innovative Smart Grid Technologies Conference, ISGT 2020

\section{Document Version:}

Peer reviewed version

Queen's University Belfast - Research Portal:

Link to publication record in Queen's University Belfast Research Portal

Publisher rights

(c) 2020 IEEE. This work is made available online in accordance with the publisher's policies. Please refer to any applicable terms of use of the publisher.

\section{General rights}

Copyright for the publications made accessible via the Queen's University Belfast Research Portal is retained by the author(s) and / or other copyright owners and it is a condition of accessing these publications that users recognise and abide by the legal requirements associated with these rights.

Take down policy

The Research Portal is Queen's institutional repository that provides access to Queen's research output. Every effort has been made to ensure that content in the Research Portal does not infringe any person's rights, or applicable UK laws. If you discover content in the Research Portal that you believe breaches copyright or violates any law, please contact openaccess@qub.ac.uk. 


\section{System Service Provision Capabilities of Storage Devices Connected to a MV Distribution Network: A Northern Ireland Case Study}

\author{
Arijit Bagchi, Robert Best and D. John Morrow \\ School of Electrical, Electronics Engineering \\ and Computer Science, Queens University Belfast \\ Belfast, United Kingdom \\ Email: bagchi.arijit@gmail.com
}

\author{
Jonathan Pollock \\ ESB Networks \\ Republic of Ireland
}

\author{
Ian Bailie and Andrew Cupples \\ Northern Ireland Electricity Networks \\ Belfast, United Kingdom
}

\begin{abstract}
Investigation of ancillary service provision capabilities of storage devices is an important area of research in the context of smart grids. This paper presents the preliminary results of a case study conducted in collaboration with Northern Ireland's distribution network operator for exploring system service provision capabilities of storage devices. Using PMU data from a local substation, the study first identifies potential voltage and/or line loading violations owing to injections from planned DG connections in an $11 \mathrm{kV}$ MV distribution network. A MATLAB based multi-period, security-constrained optimization formulation is then proposed for calculating optimal storage dispatches while minimizing DG curtailment as well as storage operation costs. The effectiveness of the proposed approach is verified by feeding back the optimization outputs into the test distribution network modelled in NEPLAN. Results indicate that both active and reactive power outputs of the storage inverters can be independently or simultaneously controlled for effectively relieving network violations.
\end{abstract}

Index Terms-Ancillary Services, Energy Storage Devices, MV Distribution Network, Renewable Energy Systems.

\section{INTRODUCTION}

Global climate change concerns have led to increased adoption of renewable and associated technologies (e.g., a mix of wind turbine generators, PV systems, storage devices and electric vehicles) in low and medium voltage (MV) distribution networks worldwide [1]. From a Northern Ireland perspective, approximately $38.6 \%$ of the total electrical energy consumed over the April 2018 - March 2019 period was generated using renewable resources [2]. The top three contributors (by energy type) in the total renewable generation pool in Northern Ireland over this period were wind at $83 \%$, followed by biogas at $6.2 \%$ and others (essentially a mix of hydro, tidal, combined heat and power as well as PV systems) at $4.3 \%$ [2]. Storage devices have also received a lot of focus in recent literatures for exploring their capabilities to offer a variety of system services (e.g., voltage regulation [3], loss reduction [4], reliability improvement [5], etc.) while allowing the deferral of traditional network reinforcement measures. This paper presents the results of a case study conducted in collaboration with Northern Ireland's distribution network operator, Northern Ireland Electricity Networks (NIEN), for investigating the potential of storage devices to offer ancillary services (e.g., voltage regulation, congestion relief, etc.) while connected to an $11 \mathrm{kV}$ MV distribution network.

In related literature, the authors in [3] and [6] propose coordinated control schemes for offering voltage regulation services using multiple energy storage devices (ESD) in a low voltage (LV) distribution network with high PV penetration. The proposed schemes consist of both distributed and localized controls for ensuring effective regulation of feeder voltages while preventing excessive saturation or depletion of individual batteries. A suitable strategy for determining optimal active and reactive power dispatches from storage inverters is presented in [4] for a three-phase, four-wire unbalanced distribution network, so as to minimize losses while satisfying network security constraints. Multi-objective optimization formulations are presented in [7] and [8] for investigating the trade-offs between distribution system and battery integration costs. While the former cost component is further broken down in terms of the different system services on offer, e.g., voltage regulation, peak shaving and loss reduction, the latter comprises depreciation, capital, operation and maintenance $(\mathrm{O} \& \mathrm{M})$ as well as energy costs pertaining to the batteries.

Using a real-life $11 \mathrm{kV}$ distribution network located in Northern Ireland as a test system, this study investigates the ability of storage devices to relieve nodal voltage and/or line loading violations in the network arising from peak distributed generator (DG) injections. The study is conducted according to the following steps.

Step 1: The model of the test distribution network (developed in NEPLAN) along with half-hourly PMU data for a week in winter 2017 are obtained from NIEN, and load flow studies (incorporating the measured data) are performed to identify potential network security violations stemming from DG injections. Step 2: A multi-timescale optimization formulation is then solved in MATLAB for obtaining the active $(P)$ and reactive $(Q)$ power dispatches from storage inverters, so as to minimize the DG curtailment as well as storage O\&M costs while ensuring that all network security constraints are satisfied. Step 3: Finally, the optimal $P \& Q$ 


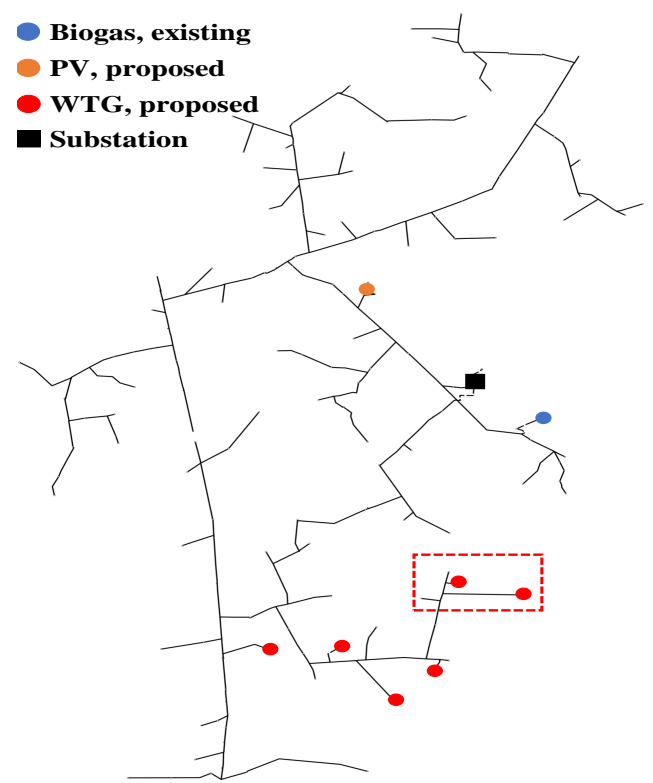

Fig. 1: Layout of $11 \mathrm{kV}$ test distribution network located in Northern Ireland

dispatch schedules obtained from MATLAB are fed back into the NEPLAN model for verifying if the identified network violations from Step 1 are indeed cleared. Results obtained from the performed simulations indicate that both active and reactive power outputs of the storage inverters can be independently or simultaneously controlled for effectively relieving network security violations.

\section{LAYOUT OF MV DistRibution NETWORK}

The layout of the $11 \mathrm{kV}$ 'test distribution network' (henceforth referred to as 'TDN') model obtained from NIEN is presented in Fig. 1. The TDN is operated as a radial network with four feeders, 227 nodes (including the reference busbar at the substation) and 226 line segments (comprising both overhead lines and underground cables). The substation (denoted by the black square in Fig. 1) houses two $33 / 11 \mathrm{kV}$ transformers which link the TDN to the upstream network. There is an existing biogas generator with an installed capacity of $3.2 \mathrm{MW}$ connected at the blue circle in Fig. 1. Additionally, there are several planned DG connections as follows. While there is a proposal to connect a $42 \mathrm{~kW}$ solar farm at the orange circle, six separate wind farms (ranging from $75 \mathrm{~kW}$ to $250 \mathrm{~kW}$ ) are planned to be connected at the red circles as shown in Fig. 1. Finally, the total installed demand associated with the TDN is 3.678 MW and 1.217 Mvar.

\section{Investigation of System Service Provision CApabilities of Storage Devices}

\section{A. Step 1: Analysis of Network Violations Incorporating Planned DG Connections Only}

For this part of the study, half-hourly PMU data corresponding to feeder current measurements at the TDN substation as well as $P$ and $Q$ injections from the existing biogas generator were obtained from NIEN for a week in winter 2017. Additionally, wind speed and solar irradiance data at half-hourly resolution for the same week for a location in Northern Ireland were obtained from [9], and the extracted data was converted to corresponding power outputs associated with the proposed wind turbine generator (WTG) and PV system connections. Several generation and load growth scenarios were considered, and load flow time simulations were performed in NEPLAN (incorporating the PMU data and proposed DG connections) for identifying potential nodal voltage and/or line loading violations in the network. Note that storage devices were not incorporated at this stage of the study.

\section{B. Step 2: Multi-Period Security-Constrained Optimization Formulation Incorporating DGs and Storage Devices}

Incorporating storage devices (along with inverters) into the network, this section presents a multi-period optimization formulation for computing $P-Q$ dispatch schedules of the inverters so as to minimize renewable generation curtailment while relieving potential network security violations (identified in Step 1). The cost-minimizing objective function (1) consists of two parts. The terms in the first line of (1) represent the cost of curtailing the active power outputs of the proposed renewable DGs. Noting that frequent charging/discharging of storage devices accelerate their depreciation, the objective function also includes an O\&M cost component for the batteries in the second line of (1). On similar lines with the approach adopted in [10], this cost is expressed as being proportional to the amount of energy being charged into or discharged from the ESDs.

$$
\begin{aligned}
\min . \sum_{t \in T} & {\left[\sum_{g \in G_{p}}\left(\lambda_{g} \cdot C_{g, t}\right)\right.} \\
& \left.+\sum_{s \in S}\left(\lambda_{s}^{c} \cdot \eta_{c} \cdot P_{s, t}^{c}\right)+\sum_{s \in S}\left(\left(\frac{\lambda_{s}^{d}}{\eta_{d}}\right) \cdot P_{s, t}^{d}\right)\right]
\end{aligned}
$$

While the set of all proposed DG connections as well as all storage devices are denoted by $G_{p}$ and $S$ respectively in (1), the set of all simulation time steps is represented by $T$. Decision variables $C_{g, t}, P_{s, t}^{c}$ and $P_{s, t}^{d}$ (all in $\mathrm{MW}$ ) respectively denote the active power curtailment of the DGs as well as the charging and discharging power at the DC link of the batteries at time $t$. The corresponding unit costs $(\$ / \mathrm{MWh})$ associated with the decision variables are respectively denoted by parameters $\lambda_{g}, \lambda_{s}^{c}$ and $\lambda_{s}^{d}$ in (1). Finally, battery charging and discharging efficiencies are denoted by parameters $\eta_{c}$ and $\eta_{d}$, respectively.

Objective function (1) is solved subject to a list of system constraints being satisfied. While the active $\left(P_{g, t}, \mathrm{MW}\right)$ and reactive $\left(Q_{g, t}\right.$, Mvar) power outputs of existing DGs satisfy the corresponding PMU measurement data, constraints (2)(3) enforce appropriate upper and lower bounds on the $P-Q$ outputs of proposed DG connections. Parameter $P_{g, t}^{W}$ (MW) denotes the active power output of the concerned DG at time $t$, as calculated using meteorological data (refer to Section 
III-A for details). While parameters $P_{g}^{R}(\mathrm{MW})$ and $Q_{g}^{R}$ (Mvar) respectively denote the nameplate $P$ and $Q$ ratings of the DGs, decision variable $Q_{g, t}$ (Mvar) represents the reactive power output of the concerned DG at time $t$.

$$
\begin{gathered}
0 \leq C_{g, t} \leq P_{g, t}^{W}, \forall g \in G_{p}, \forall t \in T \\
-\left(\frac{Q_{g}^{R}}{P_{g}^{R}}\right) \cdot\left[P_{g, t}^{W}-C_{g, t}\right] \leq Q_{g, t} \\
\leq\left(\frac{Q_{g}^{R}}{P_{g}^{R}}\right) \cdot\left[P_{g, t}^{W}-C_{g, t}\right], \forall g \in G_{p}, \forall t \in T
\end{gathered}
$$

Relevant constraints pertaining to the storage devices are expressed using (4)-(7). Parameters $\rho_{c}$ and $\rho_{d}$ (both in MW) respectively denote the maximum power that can be charged into or discharged from storage $s$ at time $t$. Variables $E_{s, t-1}$ and $E_{s, t}$ (both in MWh) denote the energy level of storage $s$ at consecutive hours $t-1$ and $t$, respectively. While $\Delta_{t}$ (hours) denotes the duration of time-step $t$, parameters $E_{s}^{R}$ (MWh), $\sigma_{\max }(\%)$ and $\sigma_{\min }(\%)$ respectively represent the batteries' rated capacities as well as their maximum and minimum permissible states-of charge. The energy conservation principle in therefore enforced through (6), while (7) applies appropriate upper and lower bounds on the storage levels for all times $t$.

$$
\begin{gathered}
0 \leq \eta_{c} \cdot P_{s, t}^{c} \leq \rho_{c}, \forall s \in S, \forall t \in T \\
0 \leq \frac{P_{s, t}^{d}}{\eta_{d}} \leq \rho_{d}, \forall s \in S, \forall t \in T \\
E_{s, t}=E_{s, t-1}-\left(\frac{P_{s, t}^{d}}{\eta_{d}} \cdot \Delta_{t}\right) \\
+\left(\eta_{c} \cdot \Delta_{t} \cdot P_{s, t}^{c}\right), \forall s \in S, \forall t \in T \\
\sigma_{\text {min }} \cdot E_{s}^{R} \leq E_{s, t} \leq \sigma_{\max } \cdot E_{s}^{R}, \forall s \in S, \forall t \in T
\end{gathered}
$$

Constraints (8)-(9) adapted from [7] are used for defining the storage inverters. Decision variables $P_{v, t}(\mathrm{MW})$ and $Q_{v, t}$ (Mvar) respectively represent the active and reactive power outputs of inverter $v$ at time $t$. Denoting inverter efficiency using $\eta_{v}$, (8) essentially relates the charging/discharging power at the DC link of the batteries (i.e., $P_{s, t}^{c}, P_{s, t}^{d}$ ) with $P_{v, t}$ while also accounting for inverter losses. Parameter $S_{v}$ represents the nameplate MVA rating of inverter $v$. It is therefore implicitly assumed through (8)-(9) that the inverters are capable of operating in all four quadrants of the $P-Q$ axes.

$$
\begin{gathered}
\left(\eta_{v} \cdot P_{s, t}^{d}\right)-\left(\frac{P_{s, t}^{c}}{\eta_{v}}\right)=P_{v, t}, \forall s, v \in S, \forall t \in T \\
P_{v, t}^{2}+Q_{v, t}^{2} \leq S_{v}^{2}, \forall v \in S, \forall t \in T
\end{gathered}
$$

Considering computational feasibility issues pertaining to modelling the 227-node TDN, line power flows and nodal voltages are expressed in (10)-(12) using the linearised (i.e., lossless) DistFlow equations originally proposed in [11] for radial distribution networks.

$$
\begin{aligned}
& P_{\ell, t}= \sum_{n \in N^{D}} P_{n, t}-\sum_{g \in G_{e}^{D}+G_{p}^{D}} P_{g, t} \\
&-\sum_{v \in S^{D}} P_{v, t}, \forall \ell \in L, \forall t \in T \\
& Q_{\ell, t}= \sum_{n \in N^{D}} Q_{n, t}-\sum_{g \in G_{e}^{D}+G_{p}^{D}} Q_{g, t} \\
&-\sum_{v \in S^{D}} Q_{v, t}, \forall \ell \in L, \forall t \in T \\
& V_{n, t}^{2}= V_{0, t}^{2}-2 \cdot \sum_{\ell \in \mathcal{L}}\left(r_{\ell} \cdot P_{\ell, t}\right) \\
&-2 \cdot \sum_{\ell \in \mathcal{L}}\left(x_{\ell} \cdot Q_{\ell, t}\right), \forall n \in N, \forall t \in T
\end{aligned}
$$

For a given line $\ell$, the set of all demands, existing DGs, proposed DGs and inverters that are downstream with respect to $\ell$ are defined in (10)-(11) using $N^{D}, G_{e}^{D}, G_{p}^{D}$ and $S^{D}$, respectively. Variables $P_{\ell, t}(\mathrm{MW}), Q_{\ell, t}$ (Mvar), $P_{n, t}$ (MW) and $Q_{n, t}$ (Mvar) respectively denote the active and reactive power flows through line $\ell$ and the active and reactive demands at node $n$ at time $t$. Assuming zero distribution network losses, equations (10)-(11) essentially model the power flow through line $\ell$ as being equal to the net total injections (from demands, generators and inverters) from all nodes that are downstream with respect to $\ell$ [11]. The square of the voltage magnitudes at nodes $n$ and 0 (reference node) at time $t$ are denoted by $V_{n, t}^{2}$ and $V_{0, t}^{2}\left(\mathrm{kV}^{2}\right)$ respectively in (12). It is assumed that the reference node voltage $V_{0, t}$ is known (i.e., regulated) for all times $t$. While the resistance and reactance associated with line $\ell$ are denoted by parameters $r_{\ell}$ and $x_{\ell}$ (both in Ohms) respectively in (12), the set of all lines that connect node $n$ to the reference node $n=0$ is represented by $\mathcal{L}$.

Finally, applicable constraints on line power flows as well as nodal voltage magnitudes are expressed in (13)-(14). Parameters $S_{\ell}, V_{n, \min }$ and $V_{n, \max }$ in the equations represent the MVA rating of line $\ell$ as well as the lower and upper voltage bounds (in $\mathrm{kV}$ ) for node $n$, respectively.

$$
\begin{gathered}
P_{\ell, t}^{2}+Q_{\ell, t}^{2} \leq S_{\ell}^{2}, \forall \ell \in L, \forall t \in T \\
V_{n, \min }^{2} \leq V_{n, t}^{2} \leq V_{n, \max }^{2}, \forall n \in N, \forall t \in T
\end{gathered}
$$

\section{Step 3: Verification of Optimization Results Using NEPLAN}

Decision variables $C_{g, t}, Q_{g, t}, P_{v, t}$ and $Q_{v, t}$ computed using the optimization formulation (1)-(14) are fed back into the NEPLAN model of TDN along with nodal demands and existing DG outputs extracted from the PMU measurements. Load flow simulations are preformed again in NEPLAN using the generation and load growth scenarios discussed in Section III-A, and it is verified whether the nodal voltage and/or line loading violations identified in Step 1 are indeed cleared after incorporating the storage devices (with inverters). 


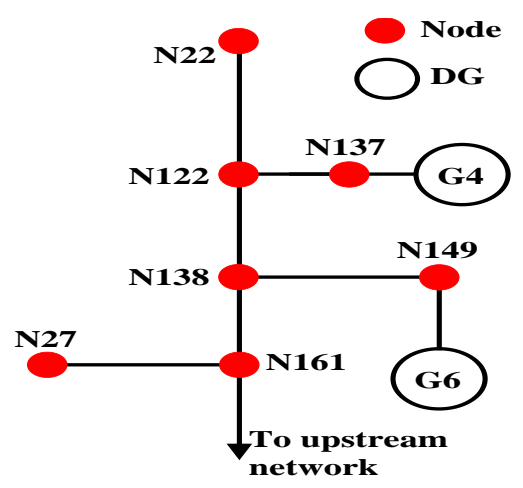

Fig. 2: Zoomed-in part of TDN with nodal voltage violations

\section{Results And Discussions}

\section{A. Step 1 Results: Identification of Network Violations}

For performing the simulations pertaining to Step 1 of the analysis, half-hourly PMU data (corresponding to feeder current measurements and $P-Q$ injections from the existing biogas generator) were obtained from NIEN for a week in winter 2017. Let the set of half-hourly loads (for all nodes of the TDN) corresponding to the obtained PMU data be designated as the 'base load scenario' (BLS). Additionally, power outputs (at half-hourly resolution) associated with the proposed DGs for the same week were extracted from [9] as mentioned in Section III-A. Let the set of half-hourly power outputs thereby obtained for the WTG and PV connections be designated as the 'base generation scenario' (BGS).

In order to analyse if the incorporation of proposed DGs cause any network security violation, several generation and load growth scenarios were considered and corresponding simulations performed in NEPLAN. Considering space restrictions, only results pertaining to the 'worst case scenario', i.e., the one associated with the worst degree of violations, will be reported for the remainder of this paper. This scenario corresponds to (proposed) DG rated capacities and nodal demands set at $120 \%$ and $100 \%$ of the respective base cases (i.e., BGS and BLS). Following are details of the violations that occurred while performing the simulation corresponding to the worst case scenario:

1) With reference to the week under study (i.e., days $1-7$ ), all violations were found to have occurred only on day 2.

2) For all time instants with violations, only some nodal voltage limits were exceeded (beyond NIEN's upper threshold of 106\%), and line loading limits were always found to be satisfied.

3) All nodal voltage violations occurred within one of the most downstream parts of the TDN with high DG penetration, as highlighted using the red dotted rectangle in Fig. 1 (a zoomed-in schematic of this area is presented in Fig. 2 for clarity sake).

The half-hourly voltage profiles of nodes $N 149$ and N137, as simulated in NEPLAN for day 2, are presented in Fig. 3. Voltages at the remaining nodes of Fig. 2 were very similar

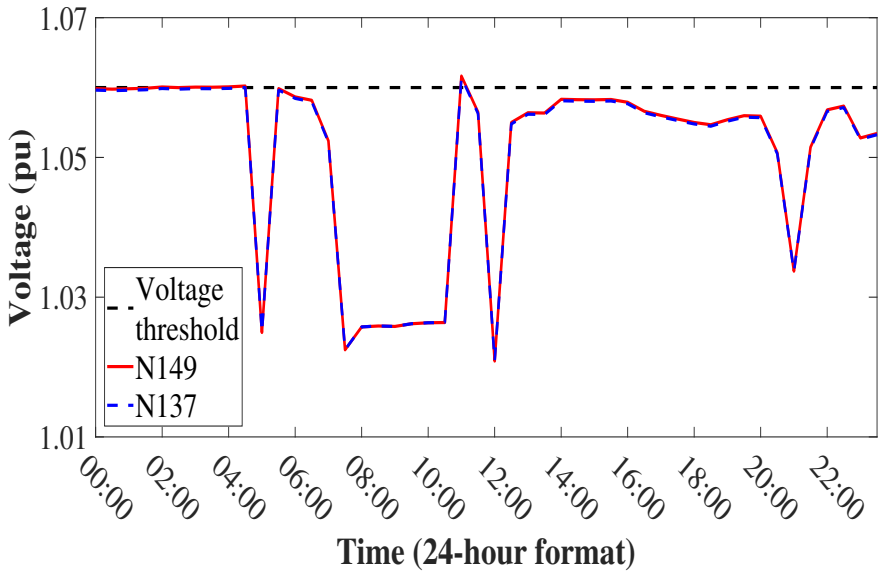

Fig. 3: Nodal voltage profiles on day 2 of week under study

(albeit slightly lower) to those reported in Fig. 3. As may be observed from the figure, nodes N149 and N137 experience voltage violations during early hours of the day owing to high WTG injections and lower demands. However, the worst voltage profile occurs at 11 am when all seven nodes of Fig. 2 experience voltages that are higher than the 1.06 per unit (pu) threshold.

\section{B. Step 2 Results: Optimal Dispatch Schedules of Storage Inverters}

For obtaining Step 2 results, it was assumed that two identical storage devices with inverters are connected at the nodes with WTGs, i.e., nodes N149 and N137 in Fig. 2. Several storage system configurations (i.e., with varying combinations of $E_{s}^{R}, \rho_{c}, \rho_{d}$ and $S_{v}$ ) were then considered for solving problem (1)-(14). Owing to space restrictions, only results pertaining to the configuration having the smallest storage/inverter size which resulted in zero DG curtailments (i.e., $C_{g, t}=0, \forall t \in T$ ) are reported in this section. Parametric values corresponding to this configuration were set as follows: $E_{s}^{R}=75 \mathrm{kWh}, \rho_{c}=\rho_{d}=37.5 \mathrm{~kW}$, and $S_{v}=61 \mathrm{kVA}$. Other parameters were assigned values as follows: $\eta_{v}=0.97, \eta_{c}=$ $\eta_{d}=0.9, \sigma_{\max }=100 \%$, and $\sigma_{\min }=20 \%, \lambda_{g}=20 \mathrm{p} / \mathrm{kWh}$ Following [10], parameters $\lambda_{s}^{c}$ and $\lambda_{s}^{d}$ were each set equal to $0.04 \mathrm{p} / \mathrm{kWh}$.

The half-hourly active $\left(P_{v, t}\right)$ and reactive $\left(Q_{v, t}\right)$ power outputs of the storage inverters computed for day 2 are presented in Fig. 4. As observed from the figure, reactive power support from the inverters (along with DGs) are sufficient to regulate nodal voltages during early hours of the day when the violations are only mild. However, when the worst voltage violations occur at 11 am (see Fig. 3), it can be observed that both active and reactive power support from the inverters is required for effectively regulating nodal voltages under the $1.06 \mathrm{pu}$ threshold. As shown in Fig. 4, inverters 1 and 2 together consume $39.96 \mathrm{~kW}$ of active power (i.e., the batteries operate in charging mode) and 115.13 kvar of reactive power at $11 \mathrm{am}$ on day 2. Note that there is negligible difference between the active and reactive power dispatch profiles of inverters 1 and 2 in Fig. 4. 


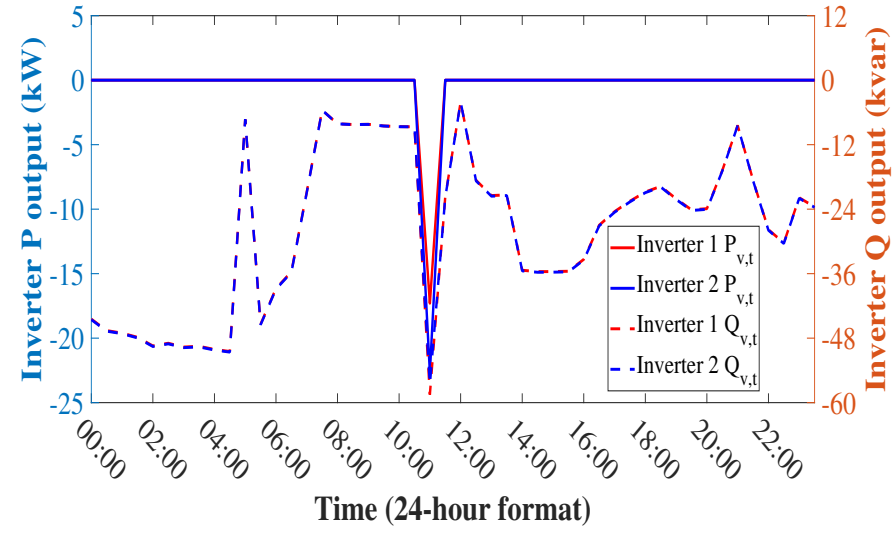

Fig. 4: Inverter power outputs on day 2 of the week under study

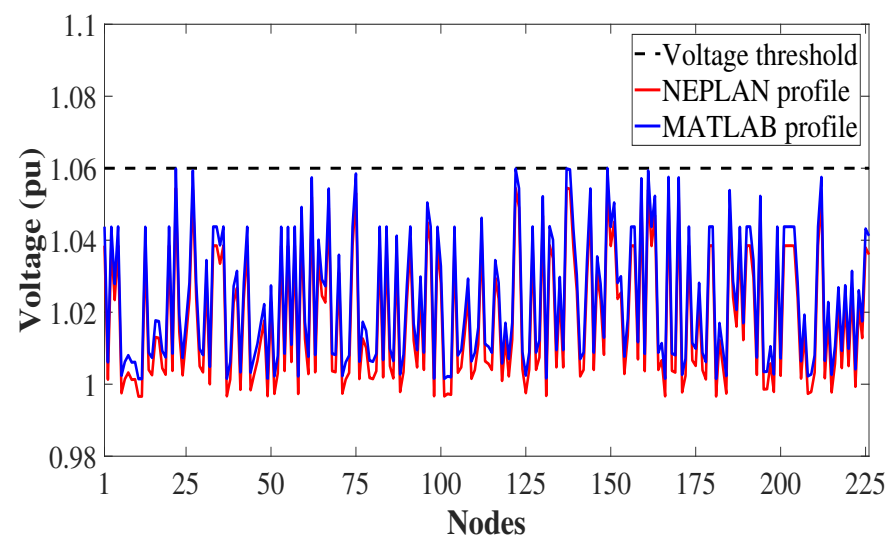

Fig. 5: TDN nodal voltage profiles at 11 am on day 2 after incorporating storage devices with inverters

\section{Step 3 Results: NEPLAN Verification}

For this final part, decision variables $P_{v, t}$ and $Q_{v, t}$ presented in Fig. 4 along with $Q_{g, t}$ (refer to (3)) are fed back into the NEPLAN model of TDN along with the PMU measurement data pertaining to nodal demands and existing DG outputs. Load flow simulations are performed for day 2 , and it is successfully verified that all nodal voltage violations reported in Section IV-A are indeed cleared as expected after solving the cost-minimization problem (1)-(14).

Once again, in the interest of brevity, only the system nodal voltages calculated (in NEPLAN) at 11 am (i.e., when the worst violations are reported in Fig. 3) on day 2 are presented in Fig. 5. Nodal voltages computed in MATLAB (solving (1)(14)) are also presented in the figure for comparison sake. As observed, all voltages are below the $1.06 \mathrm{pu}$ upper threshold after incorporation of the storage devices with inverters. Also, the voltage profiles calculated using NEPLAN and MATLAB agree reasonably well, with the MATLAB voltages always a bit higher than the NEPLAN ones (perhaps owing to utilization of the lossless DistFlow model in Section III-B). Relative errors between the two voltage profiles of Fig. 5 range from $0.43 \%-0.51 \%$.

\section{CONCLUSION}

This paper presents the results of a case study conducted for investigating potential system service provision capabilities of storage devices integrated with inverters, when connected to a MV distribution network. A multi-period optimization formulation incorporating storage devices is presented for minimizing renewable DG curtailments while satisfying network security constraints. Simulations performed on a real-life, 11 $\mathrm{kV}$ distribution network in Northern Ireland indicate that while reactive power support from the inverters and DGs are usually sufficient for regulating mild nodal voltage violations, storage devices also need to be activated (in charging mode) for time steps with more severe violations. For future studies, it would be interesting to investigate the impacts of incorporating distribution network losses and unbalance as well as renewable generation and demand uncertainty into the overall optimization problem.

\section{ACKNOWLEDGEMENT}

The authors would like to acknowledge the financial support from SPIRE2 (Storage Platform for the Integration of Renewable Energy 2) project. The SPIRE2 project is supported by the European Union's INTERREG VA Programme, managed by the Special EU Programmes Body (SEUPB). The views and opinions expressed in this document do not necessarily reflect those of the European Commission or the SEUPB.

\section{REFERENCES}

[1] A. Bagchi, L. Goel, and P. Wang, "Generation adequacy evaluation incorporating an aggregated probabilistic model of active distribution network components and features," IEEE Trans. Smart Grid, vol. 9, no. 4, pp. 2667-2680, Jul. 2018.

[2] Northern Ireland Statistics and Research Agency, Electricity consumption and renewable generation in Northern Ireland: Year ending March 2019, June 2019 (accessed July 26, 2019). [Online]. Available: https://www.economy-ni.gov.uk/sites/default/ files/publications/economy/Issue-11-Electricity-Consumption-andRenewable-Generation-in-NI-April-2018-to-March-2019.pdf

[3] M. Zeraati, M. E. H. Golshan, and J. M. Guerrero, "Distributed control of battery energy storage systems for voltage regulation in distribution networks with high PV penetration," IEEE Trans. Smart Grid, vol. 9, no. 4, pp. 3582-3593, Jul. 2018.

[4] J. D. Watson, N. R. Watson, and I. Lestas, "Optimized dispatch of energy storage systems in unbalanced distribution networks," IEEE Trans. Sustainable Energy, vol. 9, no. 2, pp. 639-650, Apr. 2018.

[5] A. Bagchi, L. Goel, and P. Wang, "Adequacy assessment of generating systems incorporating storage integrated virtual power plants," IEEE Trans. Smart Grid, vol. 10, no. 3, pp. 3440-3451, May 2019.

[6] Y. Wang, K. T. Tan, X. Y. Peng, and P. L. So, "Coordinated control of distributed energy storage systems for voltage regulation in distribution networks," IEEE Trans. Power Delivery, vol. 31, no. 3, pp. 1132-1141, Jun. 2016.

[7] J. Tant, F. Geth, D. Six, P. Tant, and J. Driesen, "Multiobjective battery storage to improve PV integration in residential distribution grids," IEEE Trans. Sustainable Energy, vol. 4, no. 1, pp. 182-191, Jan. 2013.

[8] N. Jayasekara, M. A. S. Masoum, and P. J. Wolfs, "Optimal operation of distributed energy storage systems to improve distribution network load and generation hosting capability," IEEE Trans. Sustainable Energy, vol. 7, no. 1, pp. 250-261, Jan. 2016.

[9] Centre for Environmental Data Analysis, "CEDA OGC Web Services," http://wps-web1.ceda.ac.uk/submit/choose\#.

[10] Q. Huang, Y. Xu, T. Wang, and C. Courcoubetis, "Market mechanisms for cooperative operation of price-maker energy storage in a power network," IEEE Trans. Power Systems, vol. 33, no. 3, pp. 3013-3028, May 2018.

[11] M. E. Baran and F. F. Wu, "Optimal sizing of capacitors placed on a radial distribution system," IEEE Trans. Power Delivery, vol. 4, no. 1, pp. 735-743, Jan. 1989. 